\title{
Equivalence in Alpha-Level Linear Regression
}

\author{
Jin Hee Yoon ${ }^{a}$, Hye Young Jung ${ }^{b}$, Seung Hoe Choi ${ }^{1, c}$ \\ ${ }^{a}$ School of Economics, Yonsei University; ${ }^{b}$ Department of Mathematics, Yonsei University \\ ${ }^{c}$ School of Liberal Arts and Science, Korea Aerospace University
}

\begin{abstract}
Several methods were suggested for constructing a fuzzy relationship between fuzzy independent and dependent variables. This paper reviews the use of the method by minimizing the square of the difference between an observed and a predicted fuzzy number in an $\alpha$-level linear regression model. We introduce a new distance between fuzzy numbers on the basis of a mode, a core point and a radius of an $\alpha$-level set of a fuzzy number and construct the fuzzy regression model using the proposed fuzzy distance. We also investigate sufficient conditions for an equivalence in the $\alpha$-level regression model.
\end{abstract}

Keywords: $L R$-fuzzy number, $\alpha$-level linear regression model, $\operatorname{MCR}(\alpha)$-distance, equivalence.

\section{Introduction}

Tanaka et al. (1982) were the first to introduce a fuzzy method to construct a functional relationship between fuzzy explanatory and response variables and suggested a fuzzy linear regression model as follows:

$$
Y\left(\mathbf{X}_{i}\right)=F\left(\mathbf{A}, \mathbf{X}_{i}\right), \quad i=1, \ldots, n,
$$

where $\mathbf{X}_{i}=\left(X_{i 0}, X_{i 1}, \ldots, X_{i p}\right)$ is a $(p+1)$-dimensional vector of known predictors, $\mathbf{A}=\left(A_{0}, A_{1}, \ldots, A_{p}\right)$ is a $(p+1)$-dimensional vector of unknown coefficients, $F\left(\mathbf{A}, \mathbf{X}_{i}\right)$ is a linear function about the vector $\mathbf{A}$, and $Y\left(\mathbf{X}_{i}\right)$ is a predicted variable corresponding to the input vector $\mathbf{X}_{i}$. The coefficient $A_{i}$, the predictor $X_{i p}$, and the predicted value $Y\left(\mathbf{X}_{i}\right)$ are $L R$-fuzzy numbers in the regression equation (1.1).

The membership function of an $L R$-fuzzy number $A$, denoted by $\left(a, l_{a}, r_{a}\right)_{L R}$, with a mode $a$ and a right(left) spread $r_{a}>0\left(l_{a}>0\right)$ is

$$
\mu_{A}(x)= \begin{cases}L_{A}\left(\frac{a-x}{l_{a}}\right), & \text { if } 0 \leq a-x \leq l_{a}, \\ R_{A}\left(\frac{x-a}{r_{a}}\right), & \text { if } 0 \leq x-a \leq r_{a}, \\ 0, & \text { otherwise, }\end{cases}
$$

where the functions $L$ and $R$ are continuous and strictly decreasing functions on [0,1] with $L_{A}(1)=$ $R_{A}(1)=0$ and $L_{A}(0)=R_{A}(0)=1$. In particular, if the left and right spread are same, we denote the symmetric fuzzy number as $(a, s)_{L R}$. Further, if $L_{A}(x)=R_{A}(x)=1-x$, we call the $L R$-fuzzy number a triangular fuzzy number and denote it as $\left(a, l_{a}, r_{a}\right)_{T}$. An $\alpha$-level set of the fuzzy number $A$ with the

\footnotetext{
${ }^{1}$ Corresponding author, Professor, School of Liberal Arts and Science, Korea Aerospace University, Koyang 411, South Korea. Email: shchoi@kau.ac.kr.
} 
membership function $\mu_{A}$, denoted by $A(\alpha)$, is defined by $A(\alpha)=\left\{x \in R \mid \mu_{A}(x) \geq \alpha\right\}$ for all $\alpha \in(0,1]$. The 0-level set $A(0)$ is defined as the closure of the set $\left\{x \in R \mid \mu_{A}(x)>0\right\}$.

Several methods have been suggested for constructing the fuzzy regression model (1.1). We can classify a method estimating the fuzzy regression (1.1) into two categories; the first is a numerical method minimizing the spread of the predicted fuzzy number and the second is a statistical method to minimize the sum of the distance between the observed and the predicted fuzzy numbers. Linear and non-linear programming have been used to construct the fuzzy regression model based on the objective problem with constrained conditions. The numerical methods for the fuzzy regression model was developed by many authors Chen (1999), Hojati et al. (2005), Kao and Chyu (2002), Kao and Chyu (2003), Nasrabadi and Nasrabadi (2004), Sakawa and Yano (1992), Tanaka et al. (1982). Another direction for fuzzy regression is the statistical method that minimizes the difference between the estimated and the observed fuzzy outputs. The statistical method estimates fuzzy regression models by first defining the distance between two fuzzy numbers and then generalizing to the distance within the fuzzy regression model. Many studies have been suggested for the statistical method to construct the fuzzy regression model Bargiela et al. (2007), Chang (2001), Coppi et al. (2006), Diamond (1988), Diamond and Körner (1997), D’Urso (2003), Hong and Hwang (2004), Körner and Näther (1998), Ming et al. (1997), Näther (2006), Wu (2003), Wünsche and Näther (2002), Xu and Li (2001).

In a fuzzy theory, the $\alpha$-level set $A(\alpha)$ describes the characteristics of the fuzzy number $A$ and many of the operations and relations of fuzzy numbers. Further, the membership function $\mu_{A}$ can be expressed by an indication function $\chi_{A(\alpha)}$, which has 1 at points of $A(\alpha)$ and 0 at points of $R \backslash A(\alpha)$. These imply that the predicted value $Y\left(\mathbf{X}_{i}\right)$ in the fuzzy regression (1.1) can be estimated by the $\alpha$-level set $Y_{i}(\alpha)$ of the fuzzy number based on the $\alpha$-level set of the independent and dependent variables $\left\{\left(\mathbf{X}_{i}(\alpha), Y_{i}(\alpha)\right) \mid i=1, \ldots, n\right\}$. Recently, Wu (2003) and $\mathrm{Wu}$ (2008) introduced the $\alpha$-level linear regression model and constructed the model using numerical methods.

In this paper, we construct the $\alpha$-level linear regression model of the fuzzy model (1.1) using a new fuzzy distance between fuzzy input and output numbers on the basis of the modes, core points, and radiuses of the $\alpha$-level sets of fuzzy numbers and describe an equivalence in the fuzzy least squares regression models.

The rest of this paper is organized as follows. Section 2 presents an $\alpha$-level linear regression model and we consider a new $\operatorname{MCR}(\alpha)$-distance to construct the fuzzy regression model in Section 3. The sufficient conditions for an equivalence of the $\alpha$-level linear regression model based on the eight estimation methods are suggested in Section 4 and numerical examples are given in Section 5.

\section{2. $\alpha$-Level Linear Regression Model}

This section introduces the operations of the $\alpha$-level sets of $L R$-fuzzy numbers and then suggests an $\alpha$-level linear regression model.

The $\alpha$-level set of $L R$-fuzzy number $A=\left(a, l_{a}, r_{a}\right)_{L R}$ is a closed interval as

$$
[\underline{A}(\alpha), \bar{A}(\alpha)],
$$

where $\underline{A}(\alpha)=a-l_{a} L_{A}^{-1}(\alpha)$ and $\bar{A}(\alpha)=a+r_{a} R_{A}^{-1}(\alpha)$. Thus, we can represent the $\alpha$-level set of the fuzzy number by the left and right spread as follows:

$$
A(\alpha)=\left(a, l_{A}(\alpha), r_{A}(\alpha)\right),
$$

where $l_{A}(\alpha)=l_{a} L_{A}^{-1}(\alpha)$ and $r_{A}(\alpha)=r_{a} R_{A}^{-1}(\alpha)$. Since the $\alpha$-level set of the $L R$-fuzzy number is the interval, we obtain results of the operations of the $\alpha$-level set from the interval arithmetic. In this 
paper, we let $\mathcal{F}_{L R}$ be a set of all $L R$-fuzzy numbers. The fuzzy number $A=\left(a, l_{a}, r_{a}\right)_{L R}$ is called a nonnegative if $l_{a} \leq a$.

If $A$ and $B$ are fuzzy numbers in $\mathcal{F}_{L R}$ and $\mathrm{c}$ is a real number, then the addition and multiplication for the $\alpha$-level sets $A(\alpha)$ and $B(\alpha)$ are given by

$$
\begin{aligned}
A(\alpha)+B(\alpha) & =\left(a+b, l_{A}(\alpha)+l_{B}(\alpha), r_{A}(\alpha)+r_{B}(\alpha)\right), \\
c A(\alpha) & = \begin{cases}\left(c a, c l_{A}(\alpha), c r_{A}(\alpha)\right), & \text { if } c \geq 0, \\
\left(c a,|c| r_{A}(\alpha),|c| l_{A}(\alpha)\right), & \text { if } c<0 .\end{cases}
\end{aligned}
$$

In particular, if $A$ and $B$ are the nonnegative fuzzy numbers, then the multiplication of two level sets is

$$
A(\alpha) \cdot B(\alpha)=\left(a b, a l_{B}(\alpha)+b l_{A}(\alpha)-l_{A}(\alpha) l_{B}(\alpha), a r_{B}(\alpha)+b r_{A}(\alpha)+r_{A}(\alpha) r_{B}(\alpha)\right) .
$$

Since Tanaka first introduced the fuzzy regression models in 1982, they have been applied in many fields for explaining the relation between fuzzy independent and dependent variables. The fuzzy linear regression model can be classified as follows:

(i) Input data is a fuzzy number but the regression parameter is a crisp number.

(ii) The regression parameter is a fuzzy number but the input data is a crisp number.

(iii) The input data and regression parameter are both fuzzy numbers.

The one of purposes of the fuzzy regression analysis is to estimate the fuzzy output, which has lower error between the predicted and observed output than any other estimated fuzzy number. We can do this by predicting the $\alpha$-level set of the observed fuzzy output. If the left and right reference functions $L_{A}$ and $R_{A}$ are known, we can predict the shape of the fuzzy number $A$ by some inverse images of the membership function. Further, we can use the resolution identity, suggested by Zadeh (1975), to find the membership function of the fuzzy number, in case the reference function of the fuzzy number is not known. The resolution identity states that the membership function can be rewritten as

$$
\mu_{A}(x)=\sup _{\alpha \in[0.1]} \alpha \chi_{A(\alpha)}(x) .
$$

The $\alpha$-level set of the fuzzy linear regression model, denoted by $Y\left(\mathbf{x}_{i}\right)(\alpha)$, with fuzzy output, crisp input and fuzzy parameters are

$$
\left(a_{0}, l_{A_{0}}(\alpha), r_{A_{0}}(\alpha)\right)+\left(a_{1}, l_{A_{1}}(\alpha), r_{A_{1}}(\alpha)\right) \cdot x_{i 1}+\cdots+\left(a_{p}, l_{A_{p}}(\alpha), r_{A_{p}}(\alpha)\right) \cdot x_{i p},
$$

where $0<\alpha<1$ and $x_{i j}(j=0, \ldots, p)$ are positive numbers. The operations of the $\alpha$-level set of the fuzzy number show that the above equation can be represented as follows:

$$
Y\left(\mathbf{x}_{i}\right)(\alpha)=\left(\sum_{k=0}^{p} a_{k} x_{i k}, \sum_{k=0}^{p} l_{k}(\alpha) x_{i k}, \sum_{k=0}^{p} r_{k}(\alpha) x_{i k}\right),
$$

where $x_{i 0}=1$.

Moreover, when the input and output are fuzzy numbers and the parameter is crisp we know that the $\alpha$-level set of the fuzzy linear regression model can be represented as

$$
Y\left(\mathbf{X}_{i}\right)(\alpha)=\left(\sum_{k=0}^{p} a_{k} x_{i k}, \sum_{k=0}^{p} l_{k}(\alpha) l_{X_{i k}}(\alpha), \sum_{k=0}^{p} r_{k}(\alpha) r_{X_{i k}}(\alpha)\right),
$$


where $l_{X_{i 0}}(\alpha)=r_{X_{i 0}}(\alpha)=1$ for all $\alpha$. In particular, if we assume that $a_{k}=l_{k}(\alpha)=r_{k}(\alpha)$ the equation (2.2) equals

$$
Y\left(\mathbf{x}_{i}\right)=a_{0}+a_{1} X_{i 1}+\cdots+a_{p} X_{i p} .
$$

This model was studied by Kao and Chyu (2003) and Kim et al. (2008).

Combining equations (2.1) and (2.2), we get

$$
Y\left(\mathbf{X}_{i}\right)(\alpha)= \begin{cases}\sum_{k=0}^{p} a_{k} x_{i k}, & \text { if } \alpha=1, \\ \left(\sum_{k=0}^{p} a_{k} x_{i k}, \sum_{k=0}^{p} l_{k}(\alpha) l_{X_{i k}}(\alpha), \sum_{k=0}^{p} r_{k}(\alpha) r_{X_{i k}}(\alpha)\right), & \text { if } 0<\alpha<1, \\ \left(\sum_{k=0}^{p} a_{k} x_{i k}, \sum_{k=0}^{p} l_{k} l_{X_{i k}}, \sum_{k=0}^{p} r_{k} r_{X_{i k}}\right), & \text { if } \alpha=0,\end{cases}
$$

where $x_{i 0}=1$ and $l_{X_{i 0}}(\alpha)=r_{X_{i 0}}(\alpha)=1$ for all $\alpha$.

Equations (2.1) and (2.2) are just special cases of model (2.3) when the regression coefficients $l_{k}(\alpha)$ and $r_{k}(\alpha)$ are independent of the number $\alpha$ or the parameters $l_{k}(\alpha)$ and $r_{k}(\alpha)$ are regarded as constants.

On the other hand, it is not easy to represent the the $\alpha$-level set of the fuzzy linear regression model with fuzzy input and parameters using the multiplication of the $\alpha$-level set.This is because there are many definitions for the multiplication of fuzzy numbers and the multiplication of the intervals cannot be expressed in simple terms. Therefore, in this paper, we only consider the method constructing fuzzy linear regression models with crisp input and fuzzy parameters, or fuzzy input and crisp parameters using equation (2.3).

\section{Fuzzy Regression Using MCR( $\alpha)$-Distance}

In this section, we define a new distance using the mode, core and radius of the $\alpha$-level set of the fuzzy number and then estimate the regression coefficients in the $\alpha$-level linear regression models.

Let $C_{A}(\alpha)$ and $R_{A}(\alpha)$ denote a core and radius of the $\alpha$-level set of the fuzzy number $A$. Then,

$$
C_{A}(\alpha)=a+\frac{r_{A}(\alpha)-l_{A}(\alpha)}{2} \text { and } R_{A}(\alpha)=\frac{l_{A}(\alpha)+r_{A}(\alpha)}{2} .
$$

Further, we define a distance between the $\alpha$-level sets of two fuzzy numbers $A$ and $B$, denoted by $d_{c}^{2}(A(\alpha), B(\alpha))$, as follows:

$$
d_{c}^{2}(A(\alpha), B(\alpha))=(a-b)^{2}+\left(C_{A}(\alpha)-C_{B}(\alpha)\right)^{2}+\left(R_{A}(\alpha)-R_{B}(\alpha)\right)^{2} .
$$

Since the distance includes the mode, core, and radius of the $\alpha$-level set, we call the metric $d_{c}^{2}(A(\alpha), B(\alpha)) \operatorname{MCR}(\alpha)$-distance. The simple calculation indicates that the $\operatorname{MCR}(\alpha)$-distance equals

$$
2(a-b)^{2}+(a-b)\left(r_{A}(\alpha)-r_{B}(\alpha)-l_{A}(\alpha)+l_{B}(\alpha)\right)+\frac{1}{2}\left(r_{A}(\alpha)-r_{B}(\alpha)\right)^{2}+\frac{1}{2}\left(l_{A}(\alpha)-l_{B}(\alpha)\right)^{2} .
$$

In particular, when the fuzzy numbers $A$ and $B$ are symmetric, we get

$$
d_{c}^{2}(A(\alpha), B(\alpha))=2(a-b)^{2}+\left(r_{A}(\alpha)-r_{B}(\alpha)\right)^{2} .
$$


The $\operatorname{MCR}(\alpha)$-distance gives the distance between two fuzzy numbers as follows:

$$
D(A, B)=\int_{0}^{1} \omega(\alpha) d_{c}^{2}(A(\alpha), B(\alpha)) d \alpha
$$

where $\omega(\alpha)$ is a nonnegative and continuous function on $[0,1]$.

Notice that the smaller the distance between two fuzzy numbers, the closer the $\operatorname{MCR}(\alpha)$-distance is to zero. Thus, we can estimate the fuzzy number that has a lower error between the predicted and observed fuzzy number using the $\operatorname{MCR}(\alpha)$-distance. For the normal equations about the regression coefficients, we now define the $n$-vectors and matrixes with $(p+1)$ columns and $n$ rows:

$$
\begin{aligned}
Y & =\left(y_{1}, y_{2}, \ldots, y_{n}\right)^{t}, & X & =\left[x_{i j}\right]_{n \times(p+1)}, \\
R_{Y}(\alpha) & =\left(r_{y_{1}}(\alpha), r_{y_{2}}(\alpha), \ldots, r_{y_{n}}(\alpha)\right)^{t}, & R_{X}(\alpha) & =\left[r_{x_{i j}}(\alpha)\right]_{n \times(p+1)}, \\
L_{Y}(\alpha) & =\left(l_{y_{1}}(\alpha), l_{y_{2}}(\alpha), \ldots, l_{y_{n}}(\alpha)\right)^{t}, & L_{X}(\alpha) & =\left[l_{x_{i j}}(\alpha)\right]_{n \times(p+1)},
\end{aligned}
$$

where $t$ denotes a transpose of the matrix.

When the $\alpha$-level set of fuzzy input and output numbers are given as

$$
X_{i k}(\alpha)=\left(x_{i k}, l_{x_{i k}}(\alpha), r_{x_{i k}}(\alpha)\right) \quad \text { and } \quad Y_{i}(\alpha)=\left(y_{i}, l_{y_{i}}(\alpha), r_{y_{i}}(\alpha)\right) \text {, }
$$

the least squares estimates of the coefficient of the regression equation (2.3) based on the set $\left\{\left(X_{i 1}(\alpha)\right.\right.$, $\left.\left.\ldots, X_{i p}(\alpha), Y_{i}(\alpha)\right) \mid i=1,2, \ldots, n\right\}$ and $\operatorname{MCR}(\alpha)$-distance, denoted by $\hat{P}_{c}(\alpha)=(\hat{\mathbf{a}}, \hat{\mathbf{l}}(\alpha), \hat{\mathbf{r}}(\alpha))$, are the values obtained when the quantity

$$
Q(\mathbf{a}, \mathbf{l}(\alpha), \mathbf{r}(\alpha))=\sum_{i=1}^{n} d_{c}^{2}\left(Y\left(\mathbf{X}_{i}\right)(\alpha), Y_{i}(\alpha)\right)
$$

is minimum. Here, an $i^{t h}$ term of the objective function equals

$$
\begin{aligned}
d_{c}^{2}\left(Y\left(\mathbf{X}_{i}\right)(\alpha), Y_{i}(\alpha)\right)= & 2\left(y_{i}-\sum_{k=0}^{p} a_{k} x_{i k}\right)^{2} \\
& +\left(y_{i}-\sum_{k=0}^{p} a_{k} x_{i k}\right)\left(r_{y_{i}}(\alpha)-\sum_{k=0}^{p} r_{k}(\alpha) r_{x_{i k}}(\alpha)-l_{y_{i}}(\alpha)+\sum_{k=0}^{p} l_{k}(\alpha) l_{x_{i k}}(\alpha)\right) \\
& +\frac{1}{2}\left(r_{y_{i}}(\alpha)-\sum_{k=0}^{p} r_{k}(\alpha) r_{x_{i k}}(\alpha)\right)^{2}+\frac{1}{2}\left(l_{y_{i}}(\alpha)-\sum_{k=0}^{p} l_{k}(\alpha) l_{x_{i k}}(\alpha)\right)^{2} .
\end{aligned}
$$

In order to obtain the least squares estimates $(\hat{\mathbf{a}}, \hat{\mathbf{l}}(\alpha), \hat{\mathbf{r}}(\alpha))$, we differentiate the $i^{\text {th }}$ term of the objective function partially with respect to the regression coefficients $\left(a_{0}, a_{j}, l_{0}(\alpha), l_{j}(\alpha), r_{0}(\alpha), r_{j}(\alpha)\right)$, 
and equating these partial derivative to zero, we get

$$
\begin{aligned}
\sum_{i=1}^{n} \sum_{k=0}^{p}\left(4 a_{k} x_{i k}+r_{k}(\alpha) r_{x_{i k}}(\alpha)-l_{k}(\alpha) l_{x_{i k}}(\alpha)\right) & =\sum_{i=1}^{n}\left(4 y_{i}+r_{y_{i}}(\alpha)-l_{y_{i}}(\alpha)\right), \\
\sum_{i=1}^{n} \sum_{k=0}^{p} x_{i j}\left(4 a_{k} x_{i k}+r_{k}(\alpha) r_{x_{i k}}(\alpha)-l_{k}(\alpha) l_{x_{i k}}(\alpha)\right) & =\sum_{i=1}^{n}\left[x_{i j}\left(4 y_{i}+r_{y_{i}}(\alpha)-l_{y_{i}}(\alpha)\right)\right], \\
\sum_{i=1}^{n} \sum_{k=0}^{p}\left(a_{k} x_{i k}+r_{k}(\alpha) r_{x_{i k}}(\alpha)\right) & =\sum_{i=1}^{n}\left(y_{i}+r_{y_{i}}(\alpha)\right), \\
\sum_{i=1}^{n} \sum_{k=0}^{p} r_{x_{i j}}(\alpha)\left(a_{k} x_{i k}+r_{k}(\alpha) r_{x_{i k}}(\alpha)\right) & =\sum_{i=1}^{n} r_{x_{i j}}(\alpha)\left(y_{i}+r_{y_{i}}(\alpha)\right), \\
\sum_{i=1}^{n} \sum_{k=0}^{p}\left(l_{k}(\alpha) l_{x_{i k}}(\alpha)-a_{k} x_{i k}\right) & =\sum_{i=1}^{n}\left(l_{y_{i}}(\alpha)-y_{i}\right), \\
\sum_{i=1}^{n} \sum_{k=0}^{p} l_{x_{i j}}(\alpha)\left(l_{k}(\alpha) l_{x_{i k}}(\alpha)-a_{k} x_{i k}\right) & =\sum_{i=1}^{n} l_{x_{i j}}(\alpha)\left(l_{y_{i}}(\alpha)-y_{i}\right) .
\end{aligned}
$$

The following theorem summarizes the normal equations for obtaining the regression coefficients and uses a rank of the matrix, which is the maximal number of linearly independent columns of the matrix.

Theorem 1. Suppose that the design matrix $X^{t} X$ has a full rank, and all components of the vectors $\left(X^{t} X\right)^{-1} X^{t} L_{Y}(\alpha)$ and $\left(X^{t} X\right)^{-1} X^{t} R_{Y}(\alpha)$ are positive. Then, the estimates of the $\alpha$-linear regression coefficients using the $\operatorname{MCR}(\alpha)$-distance are given by

$$
\hat{\mathbf{a}}=\left(X^{t} X\right)^{-1} X^{t} Y, \quad \hat{\mathbf{l}}(\alpha)=\left(X^{t} X\right)^{-1} X^{t} L_{Y}(\alpha) \quad \text { and } \quad \hat{\mathbf{r}}(\alpha)=\left(X^{t} X\right)^{-1} X^{t} R_{Y}(\alpha) .
$$

Proof: First, adding the first equation, -1 times the third and fifth equations in (3.1) produce

$$
\sum_{i=1}^{n} y_{i}=\sum_{i=1}^{n} \sum_{k=0}^{p} a_{k} x_{i k} .
$$

When the input $X_{i k}(k=1, \ldots, p)$ are crisp, we know that $l_{x_{i k}}(\alpha)=r_{x_{i k}}(\alpha)=x_{i k}$ in the equation (2.2). Hence, the the second, fourth, and sixth equations in (3.1) give

$$
\sum_{i=1}^{n} y_{i} x_{i j}=\sum_{i=1}^{n} x_{i j} \sum_{k=0}^{p} a_{k} x_{i k} .
$$

Equations (3.2) and (3.3) and the vector notation show that the normal equation for estimating the regression coefficients $\mathbf{a}=\left(a_{0}, \ldots, a_{p}\right)^{t}$ is

$$
X^{t} Y=\left(X^{t} X\right) \mathbf{a} .
$$

On the other hand, equation (3.2) and the third equation in (3.1) gives

$$
\sum_{i=1}^{n} \sum_{k=0}^{p} r_{k}(\alpha) x_{i k}=\sum_{i=1}^{n} r_{y_{i}}(\alpha) .
$$


Moreover, using the fourth equation in (3.1) and equation (3.3) we have

$$
\sum_{i=1}^{n} r_{y_{i}}(\alpha) x_{i j}=\sum_{i=1}^{n} x_{i j} \sum_{k=0}^{p} r_{k}(\alpha) r_{x_{i k}}(\alpha) .
$$

Thus, from the above equations, we get the normal equation as

$$
R_{X}(\alpha)^{t} R_{Y}(\alpha)=\left(R_{X}(\alpha)^{t} R_{X}(\alpha)\right) \mathbf{r}(\alpha),
$$

where $\mathbf{r}(\alpha)=\left(r_{0}(\alpha), \ldots, r_{p}(\alpha)\right)^{t}$.

Using the same methods that were used in the above normal equation, we show that the normal equation about the regression coefficient equals

$$
L_{X}(\alpha)^{t} L_{Y}(\alpha)=\left(L_{X}(\alpha)^{t} L_{X}(\alpha)\right) \mathbf{l}(\alpha),
$$

where $\mathbf{l}(\alpha)=\left(l_{0}(\alpha), \ldots, l_{p}(\alpha)\right)^{t}$. The normal equations complete the proof.

Theorem 2. Suppose that the vectors $\left(R_{X}(\alpha)^{t} R_{X}(\alpha)\right)^{-1} R_{X}(\alpha)^{t} R_{Y}(\alpha)$ have positive components and the design matrixes $X^{t} X$ and $R_{X}(\alpha)^{t} R_{X}(\alpha)$ have a full rank. If $X_{i j}$ and $Y_{i}$ are symmetric fuzzy numbers, then the estimates of the regression coefficients in equation (2.3) using the MCR( $\alpha)$-distance are given by

$$
\hat{\mathbf{a}}=\left(X^{t} X\right)^{-1} X^{t} Y \quad \text { and } \quad \hat{\mathbf{r}}(\alpha)=\left(R_{X}(\alpha)^{t} R_{X}(\alpha)\right)^{-1} R_{X}(\alpha)^{t} R_{Y}(\alpha) .
$$

Proof: The assumptions imply that

$$
l_{y_{i}}(\alpha)=r_{y_{i}}(\alpha), \quad l_{x_{i j}}(\alpha)=r_{x_{i j}}(\alpha) \quad \text { and } \quad l_{k}(\alpha)=r_{k}(\alpha) .
$$

From the above results and equation (3.1) we obtain equations (3.2) and (3.3),

$$
\sum_{i=1}^{n} \sum_{k=0}^{p} r_{k}(\alpha) r_{x_{i k}}(\alpha)=\sum_{i=1}^{n} r_{y_{i}}(\alpha)
$$

and

$$
\sum_{i=1}^{n} r_{y_{i}}(\alpha) r_{x_{i j}}(\alpha)=\sum_{i=1}^{n} r_{x_{i j}}(\alpha) \sum_{k=0}^{p} r_{k}(\alpha) r_{x_{i k}}(\alpha) .
$$

The results prove the theorem.

When the $\alpha$-linear regression does not satisfy the conditions suggested in Theorems 1 and 2, we do not obtain a unique solution for the regression coefficients in (2.3). In this case, we use an objective function as follows:

$$
\min \sum_{i=1}^{n} d_{c}^{2}\left(Y\left(\mathbf{X}_{i}\right)(\alpha), Y_{i}(\alpha)\right)
$$

subject to

$$
l_{k}(\alpha) \geq 0 \quad \text { and } \quad r_{k}(\alpha) \geq 0, \quad(k=0, \ldots, p) .
$$


The least squares estimates $(\hat{\mathbf{a}}, \hat{\mathbf{l}}(\alpha), \hat{\mathbf{r}}(\alpha))$ using $\operatorname{MCR}(\alpha)$-distance indicate that the fuzzy least squares estimate $\hat{Y}_{i}(\alpha)$ of the $\alpha$-level set $Y\left(\mathbf{X}_{i}\right)(\alpha)$ equals

$$
\hat{Y}_{i}(\alpha)=\left(\sum_{k=0}^{p} \hat{a}_{k} x_{i k}, \sum_{k=0}^{p} \hat{l}_{k}(\alpha) l_{x_{i k}}(\alpha), \sum_{k=0}^{p} \hat{r}_{k}(\alpha) r_{x_{i k}}(\alpha)\right) .
$$

In Section 5, we will show the least squares regression model based on $\operatorname{MCR}(\alpha)$-distance using equation (3.4).

\section{Equivalence in the $\alpha$-Level Linear Regression Model}

In this section, we review some distances between two $\alpha$-level sets of fuzzy numbers used in constructing the fuzzy regression models and then present the sufficient conditions for an equivalence in the $\alpha$-level linear regression models.

First of all, we can use Euclidean distance to describe the distance between fuzzy numbers since the $\alpha$-level set of the fuzzy number can be regarded as a vector in $R^{3}$. The distance between two $\alpha$-level sets $A(\alpha)$ and $B(\alpha)$ on the basis of Euclidean distance can be defined by

$$
d_{e c}^{2}(A(\alpha), B(\alpha))=(a-b)^{2}+\left(r_{A}(\alpha)-r_{B}(\alpha)\right)^{2}+\left(l_{A}(\alpha)-l_{B}(\alpha)\right)^{2} .
$$

Next, we can consider the endpoints of the closed interval to define the distance between two $\alpha$-level sets. Diamond and Körner (1997), Ming et al. (1997), Chang (2001) and Xu and Li (2001) defined the distance between fuzzy numbers using the integral of the endpoints of the $\alpha$-level set. The distance based on the endpoints can be represented by

$$
d_{e p}^{2}(A(\alpha), B(\alpha))=\omega(\alpha)(\bar{A}(\alpha)-\bar{B}(\alpha))^{2}+(\underline{A}(\alpha)-\underline{B}(\alpha))^{2} .
$$

A simple calculation shows that the distance $d_{e p}^{2}(A(\alpha), B(\alpha))$ equals

$$
2(a-b)^{2}+2(a-b)\left(r_{A}(\alpha)-r_{B}(\alpha)-l_{A}(\alpha)+l_{B}(\alpha)\right)+\left(r_{A}(\alpha)-r_{B}(\alpha)\right)^{2}+\left(l_{A}(\alpha)-l_{B}(\alpha)\right)^{2},
$$

where $\omega(\alpha)=1$ on the interval $[0,1]$.

On the other hand, Chen (1999) and Nasibov (2007) used an internally dividing point and a width of the $\alpha$-level set to measure the distance between two fuzzy numbers. We write the internally dividing point and the width of the $\alpha$-level set $A(\alpha)$ as

$$
I_{\lambda}(A(\alpha))=\lambda \underline{A}(\alpha)+(1-\lambda) \bar{A}(\alpha) \text { and } W(A(\alpha))=\bar{A}(\alpha)-\underline{A}(\alpha),
$$

where $0 \leq \lambda \leq 1$. The distance between two $\alpha$-level sets $A(\alpha)$ and $B(\alpha)$, introduced by Chen (1999), can be represented as

$$
d_{c h}^{2}(A(\alpha), B(\alpha))(\lambda)=\left(I_{\lambda}(A(\alpha))-I_{\lambda}(B(\alpha))\right)^{2}+\left(r_{A}(\alpha)-r_{B}(\alpha)\right)^{2}+\left(l_{A}(\alpha)-l_{B}(\alpha)\right)^{2} .
$$

Using the mode and spreads of the fuzzy number, the distance $d_{c h}^{2}(A(\alpha), B(\alpha))(1 / 2)$ equals

$$
\begin{aligned}
& (a-b)^{2}+\frac{5}{4}\left(r_{A}(\alpha)-r_{B}(\alpha)\right)^{2}+\frac{5}{4}\left(l_{A}(\alpha)-l_{B}(\alpha)\right)^{2} \\
& +2(a-b)\left(r_{A}(\alpha)-r_{B}(\alpha)-l_{A}(\alpha)+l_{B}(\alpha)\right)-\frac{1}{2}\left(r_{A}(\alpha)-r_{B}(\alpha)\right)\left(l_{A}(\alpha)-l_{B}(\alpha)\right) .
\end{aligned}
$$


Further, we can express the distance proposed by Nasibov (2007) as

$$
d_{n a}^{2}(A(\alpha), B(\alpha))(\lambda)=\omega(\alpha)\left[\left(I_{\lambda}(A(\alpha))-I_{\lambda}(B(\alpha))\right)^{2}+\left(W_{A}(\alpha)-W_{B}(\alpha)\right)^{2}\right] .
$$

When $\omega(\alpha)=1$ on $[0,1]$, we know that the distance $d_{n a}^{2}(A(\alpha), B(\alpha))(1 / 2)$ is equal to

$$
\begin{aligned}
& (a-b)^{2}+\frac{5}{4}\left(r_{A}(\alpha)-r_{B}(\alpha)\right)^{2}+\frac{5}{4}\left(l_{A}(\alpha)-l_{B}(\alpha)\right)^{2} \\
& +2(a-b)\left(r_{A}(\alpha)-r_{B}(\alpha)-l_{A}(\alpha)+l_{B}(\alpha)\right)+\frac{3}{2}\left(r_{A}(\alpha)-r_{B}(\alpha)\right)\left(l_{A}(\alpha)-l_{B}(\alpha)\right) .
\end{aligned}
$$

Now, we consider an equivalence of the $\alpha$-level linear regression models. Similar to $\operatorname{MCR}(\alpha)$ distance in Section 3, we can estimate the regression coefficients in (2.3) by applying the distance between $\alpha$-level sets (as suggested by many authors) to the $\alpha$-level linear regression models. In order to guarantee the solution of the normal equation, we add the distances introduced in this section to the square of the difference between the modes of the fuzzy numbers. If there exist solutions of the normal equation, the distance changed by adding the mode has the same solutions with the original distance.

We denote the value that minimizes the following objective function

$$
\sum_{i=1}^{n}\left[d_{e c}^{2}\left(Y\left(\mathbf{X}_{i}\right)(\alpha), Y_{i}(\alpha)\right)+\left(y_{i}-\sum_{k=0}^{p} a_{k} x_{i k}\right)^{2}\right]
$$

using Euclidean distance $d_{e c}^{2}\left(Y\left(\mathbf{X}_{i}\right)(\alpha), Y_{i}(\alpha)\right)$ as $\hat{P}_{e c}(\alpha)$. We also denote the estimator derived by using the endpoints as $\hat{P}_{e p}(\alpha)$, suggested by Chen (1999) as $\hat{P}_{c h}(\alpha)$, and by Nasibov (2007) as $\hat{P}_{n a}(\alpha)$.

The following theorem shows that fuzzy regression coefficients estimated by mutually different methods are the same.

Theorem 3. Suppose that the $\alpha$-linear regression model (1.1) satisfies conditions given in Theorem 1 (or Theorem 2). Then, the estimated regression coefficients $\hat{P}_{e c}(\alpha), \hat{P}_{e p}(\alpha), \hat{P}_{c h}(\alpha)$ and $\hat{P}_{n a}(\alpha)$ are all the same as the least squares estimators using the $(\hat{\mathbf{a}}, \hat{\mathbf{l}}(\alpha), \hat{\mathbf{r}}(\alpha))$ suggested in Theorem 1 (or Theorem 2).

Proof: Although it is a tedious and intricate process to find the normal equations using the distance $d_{e c}^{2}\left(Y\left(\mathbf{X}_{i}\right)(\alpha), Y_{i}(\alpha)\right), d_{e p}^{2}\left(Y\left(\mathbf{X}_{i}\right)(\alpha), Y_{i}(\alpha)\right), d_{c h}^{2}\left(Y\left(\mathbf{X}_{i}\right)(\alpha), Y_{i}(\alpha)\right)$ and $d_{n a}^{2}\left(Y\left(\mathbf{X}_{i}\right)(\alpha), Y_{i}(\alpha)\right)$, it is not difficult to obtain the same normal equation that is obtained by using $\operatorname{MCR}(\alpha)$-distance from similar methods in Theorem 1 (or Theorem 2). Hence, we dispense with the process in the proof.

The examples in Section 5 will show that the least squares estimators $\hat{P}_{e c}(\alpha), \hat{P}_{e p}(\alpha), \hat{P}_{c h}(\alpha)$ and $\hat{P}_{n a}(\alpha)$ do not conform to the estimate $\hat{P}_{c}(\alpha)$ when the conditions given in Theorem 3 do not hold on the $\alpha$-linear regression model.

\section{Numerical Examples}

In this section, we confirm the equivalence of the $\alpha$-linear regression model estimated by several different methods through four examples, and we also compare the estimates of the parameters using several distances between $\alpha$-level sets defined by different methods. We only use triangular fuzzy 
Table 1: Numerical data for in Example 1

\begin{tabular}{cccc}
\hline \hline Input & \multicolumn{3}{c}{ Output $Y_{i}(0,1)$} \\
\cline { 2 - 4 }$x_{i}$ & $y_{i}$ & $l_{y_{i}}(0.1)$ & $l_{y_{i}}(0.1)$ \\
\hline 5 & 11 & 6.3 & 7.2 \\
8 & 16 & 4.5 & 3.6 \\
11 & 18 & 2.7 & 2.7 \\
14 & 24 & 2.7 & 1.8 \\
17 & 25 & 1.8 & 1.8 \\
19 & 30 & 3.6 & 3.6 \\
22 & 31 & 3.6 & 7.2 \\
24 & 37 & 8.1 & 9.9 \\
\hline \hline
\end{tabular}

Table 2: Numerical data for in Example 2

\begin{tabular}{|c|c|c|c|c|c|}
\hline \multicolumn{3}{|c|}{ Input $X_{i}(0.1)$} & \multicolumn{3}{|c|}{ Output $Y_{i}(0.1)$} \\
\hline$x_{i}$ & $l_{x_{i}}(0.1)$ & $l_{x_{i}}(0.1)$ & $y_{i}$ & $l_{y_{i}}(0.1)$ & $l_{y_{i}}(0.1)$ \\
\hline 2 & 1.35 & 2.25 & 4 & 3.15 & 4.05 \\
\hline 3.5 & 2.7 & 3.6 & 5.5 & 4.5 & 5.4 \\
\hline 5.5 & 4.05 & 5.85 & 7.5 & 5.85 & 7.65 \\
\hline 7 & 5.85 & 6.75 & 6.5 & 5.4 & 6.3 \\
\hline 8.5 & 7.2 & 8.1 & 8.5 & 7.2 & 8.1 \\
\hline 10.5 & 8.55 & 10.35 & 8 & 6.3 & 8.1 \\
\hline 11 & 9.45 & 10.35 & 10.5 & 9 & 9.9 \\
\hline 12.5 & 10.8 & 11.7 & 9.5 & 8.1 & 9 \\
\hline
\end{tabular}

numbers and 0.1-level sets in the examples given in this section, but it is not difficult to analyze the case of $L R$-fuzzy data, because it is also easy to obtain 0.1 -level sets of $L R$-fuzzy numbers.

In the first example, explanatory variable $\left(x_{i}\right)$ is a crisp number and response variable $\left(Y_{i}\right)$ is an asymmetric fuzzy number that have different left and right spreads.

Example 1. Table 1 shows that 0.1-level sets of triangular fuzzy numbers used by Chang and Lee (1994) and Nasibov (2007).

The fuzzy regression model using Table 5.1 and $\operatorname{MCR}(0.1)$-distance, which is proposed in Section 3 , is

$$
\hat{Y}(0.1)=(4.198,3.778,2.461)+(1.272,0.026,0.151) x_{i} .
$$

From Table 1, we can easily observe that the determinant of the matrix $X^{t} X$ is 2528 and all components of $\left(X^{t} X\right)^{-1} X^{t} L_{y}(\alpha)$ and $\left(X^{t} X\right)^{-1} X^{t} R_{y}(\alpha)$ are positive numbers. Since the data given in Table 1 satisfy the conditions of Theorem 1 , we get the same estimated $\alpha$-linear regression model as $\hat{Y}(0.1)$ in (5.1) although we use different methods, such as Euclidean distance method, the method using end points of 0.1-level sets, and the method using the distance based on internally dividing points. This result shows that there is no difference in the methods presented in Section 4 under some conditions.

Example 2. Table 2 shows the 0.1-level sets of asymmetric triangular fuzzy numbers suggested by Sakawa and Yano (1992).

The regression parameters estimated by $\mathrm{MCR}(0.1)$-distance and Euclidean distance are

$$
\hat{P}_{c}(0.1)=((3.171,0.629),(2.992,0.512),(3.432,0.527))
$$

and

$$
\hat{P}_{e c}(0.1)=((3.195,0.626),(2.948,0.519),(3.455,0.524)),
$$


Table 3: Numerical data for in Example 3

\begin{tabular}{cccccc}
\hline \hline \multicolumn{2}{c}{$Y_{i}(0.1)$} & $X_{i 1}(0.1)$ & $x_{i 1}$ & & \multicolumn{2}{c}{$X_{i 2}(0.1)$} \\
\cline { 5 - 6 } \cline { 5 - 6 }$y_{i}$ & $l_{y_{i}}(0.1)$ & 6 & $x_{i 2}$ & $l_{x_{i 2}}(0.1)$ \\
\hline 41.8 & 10.53 & 7 & 4.2 & 0.81 \\
50.4 & 11.43 & 8 & 6 & 0.9 \\
49.9 & 10.89 & 9 & 5 & 0.99 \\
53.9 & 11.07 & 10 & 4 & 0.9 \\
57.7 & 8.82 & 11 & 3.6 & 0.54 \\
60.5 & 7.38 & 12 & 3 & 0.63 \\
69.1 & 7.83 & 13 & 3.5 & 0.81 \\
74.3 & 6.03 & 14 & 5.5 & 0.81 \\
84.2 & 5.76 & 15 & 8 & 0.54 \\
90.6 & 7.02 & & & 8 & 1.53 \\
\hline \hline
\end{tabular}

respectively. Moreover, the regression parameters estimated by the methods presented in the previous section are

$$
\begin{aligned}
& \hat{P}_{e p}(0.1)=((3.2,0.633),(2.936,0.529),(3.448,0.518)), \\
& \hat{P}_{n a}(0.1)=((3.199,0.626),(2.949,0.519),(3.467,0.522))
\end{aligned}
$$

and

$$
\hat{P}_{c h}(0.1)=((3.199,0.626),(2.933,0.521),(3.445,0.525)),
$$

respectively. From the above result, we can conclude that the estimated parameters are different in case the fuzzy regression model has asymmetric fuzzy input and output data. There is a difference between the regression parameters estimated by the method using the mode and the distance of $\alpha$-level sets $\hat{P}_{c}(0.1), \hat{P}_{n a}(0.1)$, and $\hat{P}_{c h}(0.1)$, and the method using the difference of the components of $\alpha$-level sets $\hat{P}_{e c}(0.1)$ and $\hat{P}_{e p}(0.1)$. This shows that we have to compare the efficiency of the different methods when the sufficient conditions given in Theorem 2 are not satisfied.

In the following example, we consider the fuzzy regression models with symmetric fuzzy input and output data.

Example 3. Kao and Lin (2005) used the data presented in Table 3 to illustrate the entropy of the fuzzy regression model.

We can easily observe that the determinant $\operatorname{det}\left(X^{t} X\right)$ is not zero and all the components of $\left(X_{2}^{t} X_{2}\right)^{-1}$ $X_{2}^{t} L_{y}(\alpha)$ are positive, where $X_{2}^{t}=\left(\begin{array}{ccc}1 & \cdots & 1 \\ l_{x_{12}} & \cdots & l_{x_{n 2}}\end{array}\right)$. Further, the regression parameters estimated by $\operatorname{MCR}(0.1)$-distance are

$$
\hat{P}_{c}(0.1)=((3.667,4.916,1.699),(7.924,0.889)) .
$$

We verified that the estimates presented in Section $4 \hat{P}_{e c}(0.1), \hat{P}_{e p}(0.1), \hat{P}_{n a}(0.1)$, and $\hat{P}_{c h}(0.1)$ are the same as $\hat{P}_{c}(0.1)$ in (5.2) through simple computations using Matlab. This result coincides with Theorem 2. So, if we want to construct the fuzzy regression models, it is sufficient to choose a special method similar to Example 1.

The next example presents the use of restricted conditions in cases where the predicted regression coefficients have negative spreads.

Example 4. Kim and Bishu (1998) introduced the least squares method using the mode and the distance of $\alpha$-level sets, and they used real data presented in Table 4 to estimate the fuzzy multiple regression model. 
Table 4: Numerical data for in Example 4

\begin{tabular}{|c|c|c|c|c|}
\hline \multicolumn{2}{|c|}{$Y_{i}(0.1)$} & \multicolumn{3}{|c|}{$X_{i}$} \\
\hline$y_{i}$ & $l_{y_{i}}(0.1)$ & $x_{i 1}$ & $x_{i 2}$ & $x_{i 3}$ \\
\hline 5.83 & 3.04 & 2 & 0 & 15.25 \\
\hline 0.85 & 0.468 & 0 & 5 & 14.13 \\
\hline 13.9 & 7.65 & 1.13 & 1.5 & 14.13 \\
\hline 4 & 2.25 & 2 & 1.25 & 13.63 \\
\hline 1.65 & 0.909 & 2.19 & 3.75 & 14.75 \\
\hline 1.58 & 0.864 & 0.25 & 3.5 & 13.75 \\
\hline 8.18 & 4.491 & 0.75 & 5.25 & 15.25 \\
\hline 1.85 & 1.017 & 4.25 & 21 & 3.5 \\
\hline
\end{tabular}

Although the design matrix $X^{t} X$ is nonsingular, the three components of the estimated coefficients $\left(X^{t} X\right)^{-1} X^{t} L_{y}(\alpha)$ are negative, and only one component of the vector is positive. Hence, we should consider the restricted conditions where the spreads are positive. The regression parameter estimated by using the objective function (5.1) with constraints is

$$
\hat{P}_{c}(0.1)=((-16.8,-1.1,-1.18,1.856),(0,0,0,0.184)) .
$$

We confirmed that if we apply the restricted conditions $l_{k}(\alpha) \geq 0$ and $r_{k}(\alpha) \geq 0(k=0, \ldots, p)$ to every method presented in Section 4 , the results are the same as $\hat{P}_{c}(0.1)$ in (5.3) using Matlab programming.

\section{Conclusions}

In this paper, we verified that if the $\alpha$-linear regression model is used, we can express the relation between the variables that cannot be expressed clearly due to the vagueness of the data. Then, we introduced $\operatorname{MCR}(\alpha)$-distance based on the core and the radius of the $\alpha$-level sets of predicted fuzzy numbers and observed fuzzy numbers. We also proposed the sufficient conditions for algebraically expressing the regression parameters of the $\alpha$-linear regression model that are estimated by $\operatorname{MCR}(\alpha)$ distance method. Further, we confirmed that the eight estimators are equivalent in the following two cases: crisp input-fuzzy output and symmetric fuzzy input-output case. We verified that if the data satisfy the sufficient conditions proposed in this paper, we can choose only one method to estimate the regression parameters. Although we used computer programs because the equations are not algebraically solvable when we use $\operatorname{MCR}(\alpha)$-distance method in the case of asymmetric fuzzy input-output data. We confirmed this by the example that the results are different from those obtained using the Euclidean method.

Further research is needed to find the condition for raising the efficiency of each estimation method to the optimal level when the regression coefficients estimated by eight methods introduced in this paper are not equivalent.

\section{References}

Bargiela, A., Pedrycz, W. and Nakashima, T. (2007). Multiple regression with fuzzy data, Fuzzy Sets and Systems, 158, 2169-2188.

Chang, P.-T. and Lee, E. S. (1994). Fuzzy least absolute deviations regression and the conflicting trends in fuzzy parameters, Computers \& Mathematics with Applications, 28, 89-101.

Chang, Y. (2001). Hybrid fuzzy least-squares regression analysis and its reliability measures, Fuzzy Sets and Systems, 119, 225-246. 
Chen, Y.-S. (1999). Fuzzy ranking and quadratic fuzzy regression, Computers \& Mathematics with Applications, 38, 265-279.

Coppi, R., D’Urso, P., Giordani, P. and Santoro, A. (2006). Least squares estimation of a linear regression model with $L R$ fuzzy response, Computers \& Mathematics with Applications, 51, 267-286.

Diamond, P. (1988). Fuzzy Least Squares, Information Sciences: An International Journal, 46, 141157.

Diamond, P. and Körner, R. (1997). Extended fuzzy linear models and least squares estimates, Computers \& Mathematics with Applications, 33, 15-32.

D’Urso, P. (2003). Linear regression analysis for fuzzy/crisp input and fuzzy/crisp output data, Computers \& Mathematics with Applications, 42, 47-72.

Hojati, M., Bector, C. R. and Smimou, K. (2005). A simple method for computation of fuzzy linear regression, European Journal of Operational Research, 166, 172-184.

Hong, D. H. and Hwang, C. (2004). Extended fuzzy regression models using regularization method, Information Sciences: An International Journal, 164, 31-46.

Kao, C. and Chyu, C. (2002). A fuzzy linear regression model with better explanatory power, Fuzzy Sets and Systems, 126, 401-409.

Kao, C. and Chyu, C. (2003). Least-squares estimates in fuzzy regression analysis, European Journal of Operational Research, 148, 426-435.

Kao, C. and Lin, P. (2005). Entropy for fuzzy regression analysis, International Journal of Systems Science, 36, 869-876.

Kim, B. and Bishu, R. R. (1998). Evaluation of fuzzy linear regression models by comparing membership functions, Fuzzy Sets and Systems, 100, 343-352.

Kim, H. K., Yoon, J. H. and Li, Y. (2008). Asymptotic properties of least squares estimation with fuzzy observations, Information Sciences: An International Journal, 178, 439-451.

Körner, R. and Näther, W. (1998). Linear regression with random fuzzy variables: Extended classical estimates, best liner estimates, least squares estimate, Journal of Information Science, 109, 95118.

Ming, M., Friedman, M. and Kandel, A. (1997). General fuzzy least squares, Fuzzy Sets and Systems, 88, 107-118.

Nasibov, E. N. (2007). Fuzzy least squares regression model based of weighted distance between fuzzy numbers, Automatic Control and computer sciences, 41, 15-26.

Nasrabadi, N. N. and Nasrabadi, E. (2004). A mathematical-programming approach to fuzzy linear regression analysis, Applied Mathematics and Computation, 155, 873-881.

Näther, W. (2006). Regression with fuzzy random data, Computational Statistics \& Data Analysis, 51, 235-252.

Sakawa, M. and Yano, H. (1992). Multiobjective fuzzy linear regression analysis for fuzzy inputoutput data, Fuzzy Sets and Systems, 47, 173-181.

Tanaka, H., Uejima, S. and Asai, K. (1982). Linear regression analysis with fuzzy model, IIEEE Transactions On Systems, Man, And Cybernet, 12, 903-907.

Wu, H.-C. (2003). Fuzzy estimates of regression parameters in linear regression models for imprecise input and output data, Computational Statistics \& Data Analysis, 42, 203-217.

Wu, H.-C. (2008). Fuzzy linear regression model based on fuzzy scalar product, Soft Computing, 12, 469-477.

Wünsche, A. and Näther, W. (2002). Least-squares fuzzy regression with fuzzy random variables, Fuzzy Sets and Systems, 130 , 43-50. 
$\mathrm{Xu}, \mathrm{R}$. and Li, C. (2001). Multidimensional least-squares fitting with a fuzzy model, Fuzzy Sets and Systems, 119, 215-223.

Zadeh, L. A. (1975). The concept of linguistic variable and its application to approximate reasoning I, Information Sciences: An International Journal, 8, 199-249. 